DOI: $10.21802 / \mathrm{artm} .2019 .2 .10 .113$.

УДК 616-072+616.12-008.46

\title{
LABORATORY DIAGNOSTICS OF EARLY HEART FAILURE
}

\author{
N.B. Tymochko \\ Ivano-Frankivsk National Medical University, Department of Internal Medicine №2 and Nursing, \\ Ivano-Frankivsk, Ukraine, \\ ORCID ID: 0000-0002-5319-5468, \\ e-mail:dr.ntymochko@gmail.com
}

\begin{abstract}
Many diseases of the cardiovascular system require precisely timely diagnosis to prevent acute and chronic pathological processes and complications. The article presents laboratory indicators and markers, the use of which helps a modern physician to diagnose correctly and timely, predict the onset of illness in patients and monitor the treatment of a diagnosis such as heart failure.

Confirmation of $\mathrm{CH}$ and/or cardiac dysfunction by echocardiography is mandatory and should be carried out as soon as possible after suspected $\mathrm{CH}$.

Electrocardiographic examination (ECG) should be performed for each patient with suspected CH. If ECG is completely normal, $\mathrm{CH}$, especially systolic dysfunction, is unlikely $(<10 \%)$. X-ray examination of the chest organs is a necessary component in diagnostic $\mathrm{CH}$ search. It allows you to assess pulmonary stagnation or to show important pulmonary or thoracic causes of shortness of breath. Patients in whom resting echocardiography does not provide adequate information and in patients with suspected CHD, a further non-invasive study may include a magnetic resonance imaging (MRD) of the heart, CT (CT) of the heart, or radionuclide ventriculography.

There is evidence to support the effectiveness of using natriuretic peptides for diagnosis, addressing the issue of hospitalization / discharge and the definition of individual prognosis, and there is a lack of evidence of their use for surveillance and regulation of drug therapy. The response of healthy heart tissue to damage or mechanical stress includes the production and binding of interleukin-33 (IL-33) to ST2L by launching a cardioprotective signalling phase for the prevention of fibrosis, heart remodelling and heart failure $(\mathrm{CH})$. sST2 blocks the cardioprotective effect of IL33. Determination of ST2 at discharge can significantly reduce cases of rhizospitalization within 30 days. The level of $\mathrm{BNP}$ at $\mathrm{ST} 2>35 \mathrm{ng} / \mathrm{ml}$ is both elevated and normal, which may mislead physicians.

Often in clinical practice, doctors meet such concepts as "false-negative" and "false-positive" result, qualitative or semi-quantitative false result. This happens first through the right selection of the method of determining one or another marker. For example, in order to monitor the management of acute myocardial infarction, it is only necessary to determine the level of troponin quantitatively, since only in this case it will be seen how its numerical parameters change at the stages of treatment.

Since ST2 does not depend on factors such as BMI, gender, age, smoking status, and the presence of concomitant pathologies, it may be one of the "strongest" predictive markers of $\mathrm{CH}$. The introduction of ST2 into routine practice will significantly reduce hospital costs (reducing the number of repeated hospitalizations, eliminating unnecessary drugs, etc.) and improving the quality of life of patients with $\mathrm{CH}$ (to reduce the rate of progression of $\mathrm{CH}$ ). Based on the above, it can be accurately said that the earliest diagnostic marker of heart failure is a marker - ST2.
\end{abstract}

Keywords: laboratory diagnostic methods, heart failure, marker for heart failure, ST-2.

Introduction. Significant increasing of observed nowadays comorbid pathology in different organs and systems attract attention of researchers as well as practical physicians. A particular attention is drawn to cardiovascular diseases.

According to the WHO, Ukraine is a country №1 in Europe and №2 in the world in cardiovascular mortality rates [6]. When European countries already combat this problem actively, then in Ukraine this is a cause of $67 \%$ deaths as before. Each second Ukrainian faces cardiovascular diseases. Last year CVD took lives of 392 thousand Ukrainians [8].

On 29 September, World Heart Day takes place in order to attract attention to CVD problem, the main cause of population morbidity in the world. Each year more than 17 millions around the world die from CVD, more than from oncological diseases, respiratory diseases and diabetes all combined. A great weight of such deaths falls on countries with people of middle and modest social background [7].

Each year it is recorded about 50 thousand infarction causes in Ukraine, and what is interesting, each person of Ukraine could decrease this number significantly, if his/her efforts were directed at measurements to prevent CVD. Main risk factors of cardiac pathology include unhealthy nourishment, smoking, excess pounds, inactive lifestyle, delayed diagnostics, disregard of annual preventive examinations etc. One of main tasks of this century general physicians is preventive medicine. It is necessary to inform people of relevance of timely preventive examinations (especially when it concerns cardiovascular pathology). It is necessary also to tell about sites, which have collections of files, documents of evidencebased medicine on prevention of cardiovascular diseases, for example, the site of the program «Live with the Heart» developed together with Center for Pediatric Car- 
diology and Cardiac Surgery of $\mathrm{MOH}$ of Ukraine [9]. Such information and newly acquired knowledge will help to get patient's trust faster that in its turn will contribute to diagnostics or prevention of his problems, especially when it concerns heart failure (HF).

Aim of the research. To determine and study the most informative methods of laboratory diagnostics of heart failure.

Results of the research and discussion on them. Validation of HF and/or cardiac dysfunction with the help of echocardiography is mandatory and has to be done as fast as possible after HF has been suspected. Echocardiography is a widely accessible, fast, noninvasive and safe method that provides comprehensive infor- mation on anatomy of the heart (volumes, geometry, and weight), walls movement and valves activity. The research gives necessary information on etiology of HF.

LVEF index is the most practical meaning for specification of ventricular function to differentiate the patients with systolic dysfunction and the patients with preserved systolic function має показник ФВЛШ (in norm $>45-50 \%$ ). This limit is somewhat undefined. LVEF is not synonymic with stoke work index that depends clearly on volumes, preload, afterload, heart rate and valves activity. Systolic output can be maintained by means of heart dilation and increasing of volumes (table 1.).

Table 1

\begin{tabular}{|l|c|c|}
\hline \multirow{2}{*}{\multicolumn{1}{|c|}{ Evaluation }} & \multicolumn{2}{c|}{ Diagnosis of Heart Failure } \\
\cline { 2 - 3 } & Confirms by presence & $\begin{array}{c}\text { Denies in case of norm or ab- } \\
\text { sence }\end{array}$ \\
\hline Complete symptoms & ++ & ++ \\
\hline Complete signs & ++ & +++ \\
\hline Cardiac dysfunction on echo & +++ & ++ \\
\hline Response to therapy & +++ & ++ \\
\hline ECG & & + \\
\hline Norm & & + \\
\hline Pathology & ++ & + \\
\hline Dysrhythmia & +++ & + \\
\hline Renal dysfunction & + & + \\
\hline Chest X-ray & +++ & ++ \\
\hline Lung congestion & +++ & + \\
\hline Decreased physical power & + & + \\
\hline Pathologic pulmonary function tests & +++ & + \\
\hline Pathologic hemodynamics at rest & & \\
\hline Notes: 4 - & + & + \\
\hline
\end{tabular}

Notes: «+» - mildly; «++» - moderately; «+++» - significantly.

Electrocardiographic examination (ECG) has to be performed on each patient with suspected HF. However, a pathologic ECG has a low prognostic level for presence of HF. If the EKГ is fully normal, HF especially systolic dysfunction is unlikely $(<10 \%)$.

A chest $\mathrm{X}$-ray is a necessary component in diagnostic search of HF. It allows evaluating lung congestion or showing significant lung or thoracic causes of dyspnea. The chest X-ray (in two views) can help to find cardiomegaly, lung congestion, and fluid accumulation in pleural cavity or to show presence of lung disease or infection, which caused or worsened dyspnea. 3a виключенням застоюЕхcluding congestion, other findings may predict presence of HF only in the context of typical signs and symptoms. Cardiomegaly can be absent not only by acute, but also by chronic HF. So, on the early stages of HF this diagnostic method is uninformative too.

In patients, whose echocardiography at rest does not give an adequate information, and in patients with suspected IHD further noninvasive examination may include magnetic resonance imaging (MRI) of the heart, computer tomography (CT) of the heart or radionuclide ventriculography.

Cardiac catheterization is not necessary for routine diagnostics and treatment of patients with HF. Invasive examinations are often indicated for clarification of etiology, receiving of important prognostic information and, if considered, revascularization.

Coronary angiography in patients with HF has to be considered, when the anamnesis contains angina pectoris or ischemic LV dysfunction after cardiac arrest is suspected in those, who has notable risk factors for IHD; it may be required immediately in some patients with severe HF (shock or acute pulmonary edema) and in patients not responding to treatment adequately. Coronary angiography and LV ventriculography are also indicated for patients with refractory HF of unknown etiology and in patients with proved severe mitral regurgitation or aortic valves disease that can be potentially corrected with surgical treatment. As long as this method is invasive, so practically is not used in preventive medicine and on early or minimal clinical manifestation of CVD [4, 5].

Catheterization of right chambers of the heart provides valuable information about hemodynamics, namely filling pressure, vascular resistance and cardiac output. Its part is limited in diagnostics of HF in clinical practice. The method is used for Forrester classification and is the most precise for evaluation of hemodynamics in refractory to treatment patients before transplantation or in clinical studies evaluating invasion. Observation of hemodynamic indices through a catheter in pulmonary artery (PAC) may be considered for hospitalized patients with cardiogenic/noncardiogenic shock or for observation 
of treatment in patients with severe HF, who do not respond to therapy. However, improvement of results after PAC was not indicated.

Specific myocardial disorders may be diagnosed with the help of endomyocardial biopsy (EMB). Clinical decision has to be based on available researches causecontrol and experts' thoughts. Recently published united consensus of AHA/ACC/ESC concerning indications for EMB proved that the procedure has to be considered in patients with acute or fulminant $\mathrm{HF}$ of unknown genesis that worsens quickly as a result of ventricular arrhythmia and/or AV-block, or in patients not responding to traditional therapy of HF. EMB may also be used by chronic HF with expected infiltrative processes, such as amyloid disease, sarcoidosis, and hemochromatosis, as well as eosinophilic endocarditic and restrictive cardiomyopathy of unknown genesis [1].
Laboratory examinations. Routine diagnostic examination of patients with suspected HF includes total blood count (hemoglobin, leucocytes and platelets), plasma electrolytes, serum creatinine, evaluation of glomerular filtration rate (GFR), glucose, liver panel and urinalysis (tables 2, 3). Nevertheless, these tests are not of great significance for diagnosis by untreated mild and moderate HF. However, mild anemia, hyponatremia, hyperkalemia and worsened renal function may be observed, especially in patients that were treated with diuretics and ACE inhibitors/BAS/aldosterone antagonists. Respective laboratory observation is necessary during beginning, titration and monitoring of patients receiving medications for treatment of already proven diagnosis of HF (table 2).

Frequent pathology occurring during laboratory examination in patients with $\mathrm{HF}$

\begin{tabular}{|c|c|c|}
\hline Pathology & Cause & Clinical significance \\
\hline $\begin{array}{l}\text { Increased serum creatinine } \\
\text { level }\end{array}$ & $\begin{array}{l}\text { Renal disease, ACE inhibitor, BAS, } \\
\text { aldosterone antagonist. }\end{array}$ & $\begin{array}{l}\text { To calculate GFR. To consider lowering of } \\
\text { ACE inhibitor/BAS/aldosterone antagonist } \\
\text { dose. To check potassium and blood urea } \\
\text { nitrogen. }\end{array}$ \\
\hline $\begin{array}{l}\text { Anemia }(13 \mathrm{~g} / \mathrm{dL} \text { in males, } \\
12 \mathrm{~g} / \mathrm{dL} \text { in females })\end{array}$ & $\begin{array}{l}\text { Chronic HF, hemodilution, iron loss or } \\
\text { poor utilization, renal failure, chronic } \\
\text { disease. }\end{array}$ & Diagnostic search. To consider treatment. \\
\hline $\begin{array}{l}\text { Hyponatremia } \\
(<135 \mathrm{mmol} / \mathrm{L})\end{array}$ & $\begin{array}{l}\text { Chronic HF, hemodilution, arginine- } \\
\text { vasopressin excretion, diuretics. }\end{array}$ & $\begin{array}{l}\text { To consider lowering of diuretics dose. } \\
\text { Ultrafiltration, vasopressin antagonists. }\end{array}$ \\
\hline $\begin{array}{l}\text { Hypernatremia } \\
(>150 \mathrm{mmol} / \mathrm{L})\end{array}$ & Hyperkalemia. Dehydration. & $\begin{array}{l}\text { To evaluate water consumption. Diagnostic } \\
\text { search. }\end{array}$ \\
\hline $\begin{array}{l}\text { Hypokalemia } \\
(<3.5 \mathrm{mmol} / \mathrm{L})\end{array}$ & Diuretics, secondary aldosteronism. & $\begin{array}{l}\text { Risk of arrhythmia. To consider potassium, } \\
\text { ACE inhibitors/BAS/aldosterone antago- } \\
\text { nists use. }\end{array}$ \\
\hline $\begin{array}{l}\text { Hyperkalemia } \\
(>5.5 \mathrm{mmol} / \mathrm{L})\end{array}$ & $\begin{array}{l}\text { Renal failure, potassium and ACE in- } \\
\text { hibitors use. }\end{array}$ & $\begin{array}{l}\text { To stop treatment with potassium-sparing } \\
\text { drugs (ACE inhibitors/BAS/aldosterone } \\
\text { antagonists). To evaluate renal function and } \\
\text { pH. Risk of bradycardia. }\end{array}$ \\
\hline $\begin{array}{l}\text { Hyperglycemia } \\
(>6.5 \mathrm{mmol} / \mathrm{L})\end{array}$ & Diabetes mellitus, insulin resistance. & $\begin{array}{l}\text { To evaluate hydration, to treat glucose in- } \\
\text { tolerance. }\end{array}$ \\
\hline $\begin{array}{l}\text { Hyperuricemia } \\
(>500 \mathrm{mcmol} / \mathrm{L})\end{array}$ & $\begin{array}{l}\text { Treatment with diuretics, gout, malig- } \\
\text { nant tumor, }\end{array}$ & $\begin{array}{l}\text { Allopurinol. } \\
\text { Lowering of diuretics dosing. }\end{array}$ \\
\hline $\begin{array}{l}\mathrm{BNP}>400 \mathrm{pg} / \mathrm{ml}, \\
\text { NT-proBNP }>2000 \mathrm{pg} / \mathrm{ml}\end{array}$ & Increased stretching of ventricular wall. & $\begin{array}{l}\text { Probable HF. Indications for echo. To con- } \\
\text { sider treatment. }\end{array}$ \\
\hline $\begin{array}{l}\mathrm{BNP}<100 \mathrm{pg} / \mathrm{ml}, \\
\mathrm{NT} \text {-proBNP }<400 \mathrm{pg} / \mathrm{ml}\end{array}$ & Normal stretching of ventricular wall. & $\begin{array}{l}\text { To reconsider diagnosis. HF is unlikely, in } \\
\text { case it was not treated. }\end{array}$ \\
\hline $\begin{array}{l}\text { High albumins level } \\
(>45 \mathrm{~g} / \mathrm{L})\end{array}$ & Dehydration, myeloma. & Rehydration. \\
\hline $\begin{array}{l}\text { Low albumins level } \\
(<30 \mathrm{~g} / \mathrm{L})\end{array}$ & Inadequate nutrition, loss by kidneys. & Diagnostic search. \\
\hline $\begin{array}{l}\text { Increased } \quad \text { transaminase } \\
\text { levels }\end{array}$ & $\begin{array}{l}\text { Impaired liver function. Right ventricular } \\
\text { HF. Toxic influence of medications. }\end{array}$ & $\begin{array}{l}\text { Diagnostic search. } \\
\text { Liver congestion. } \\
\text { To reconsider treatment. }\end{array}$ \\
\hline Increased troponin levels & $\begin{array}{l}\text { Myocyte necrosis, long-term ischemia, } \\
\text { severe } \mathrm{HF} \text {, myocarditis, sepsis, renal } \\
\text { failure, PE }\end{array}$ & $\begin{array}{l}\text { To evaluate increasing stage. (often insignifi- } \\
\text { cant increasing by severe HF). Coronary } \\
\text { angiography. To decide a question on revas- } \\
\text { cularization. }\end{array}$ \\
\hline
\end{tabular}

Troponins. It is necessary to take blood samples for troponins I and $\mathrm{T}$ while suspecting HF by a clinical picture of acute coronary syndrome (ACS). Increased cardiac troponins levels indicate myocyte necrosis, so when there are any indications, it is necessary to consider possibility of revascularization and to perform necessary 
diagnostic procedures. Increased troponins levels are revealed by acute myocarditis too. Insignificantly increased cardiac troponins levels are often observed by severe HF or during decompensation in patients without proven myocardial ischemia because of ACS or in such situation, as sepsis. Increased troponins levels are a significant prognostic marker by HF, especially combined with increased natriuretic peptides. However, there is a minus; it is uninformative on early stages of HF (table 3 ).

Laboratory indices used for diagnostics of cardiovascular pathologies

Table 3

\begin{tabular}{|c|c|c|}
\hline Indices & Level in blood increases by: & Level in blood decreases by: \\
\hline LDH & $\begin{array}{l}\text { - myocardial infarction (in } 24-32 \mathrm{~h} \text { ); } \\
\text { - pulmonary infarction (in } 24 \mathrm{~h} \text { after initial pain); } \\
\text { - congestive heart failure; } \\
\text { - shock states; } \\
\text { - hypoxias; } \\
\text { - hypotensions; } \\
\text { - renal infarction. }\end{array}$ & $\begin{array}{l}\text { - specific reaction to the specific cytostatic } \\
\text { chemotherapy. }\end{array}$ \\
\hline СРК-МВ & $\begin{array}{l}\text { - myocardial infarction; } \\
\text { - severe myocarditis; } \\
\text { - after an open surgery on the heart; } \\
\text { - electrical defibrillation; } \\
\text { - cardiomyopathies; } \\
\text { - Duchenne muscular dystrophy; } \\
\text { - poly-, dermatomyositis; } \\
\text { - muscle injury; } \\
\text { - myalgias; } \\
\text { - malignant hyperthermia; }\end{array}$ & $\begin{array}{l}\text { - tumor metastases causing decrease of mus- } \\
\text { cular mass; } \\
\text { - treatment with corticosteroids; } \\
\text { - alcoholic liver disease; } \\
\text { - excessive ultraviolet radiation; } \\
\text { - colagenoses. }\end{array}$ \\
\hline Potassium & $\begin{array}{l}\text { - fast drug introduction; } \\
\text { - massive hemolysis; } \\
\text { - tissue ischemia; } \\
\text { - shock states; } \\
\text { - chronic renal failure; }\end{array}$ & $\begin{array}{l}\text { - lack of potassium supply (diets); } \\
\text { - frequent vomiting; } \\
\text { - profuse diarrheas; } \\
\text { - Crohn's disease; } \\
\text { - infusion of ACTH, cortisone, aldosterone, } \\
\text { corticosteroids; } \\
\text { - pancreatic tumors. }\end{array}$ \\
\hline Sodium & $\begin{array}{l}\text { - hypertonic dehydration; } \\
\text { - desudation; } \\
\text { - long-term dyspnea; } \\
\text { - excessive introduction of sodium salts (hyper- } \\
\text { tension). }\end{array}$ & $\begin{array}{l}\text { - vomiting, diarrhea; } \\
\text { - acute adrenal insufficiency; } \\
\text { - acute renal failure; } \\
\text { - diuretic overdose. }\end{array}$ \\
\hline CRP & $\begin{array}{l}\text { - MI (on the } 2^{\text {nd }} \text { day); } \\
\text { - transplant rejection; } \\
\text { - connective tissue diseases; } \\
\text { - extensivity of MI; } \\
\text { - recurrent case of } \mathrm{MI} \\
\text { - sepsis; } \\
\text { - meningitis. }\end{array}$ & \\
\hline $\begin{array}{l}\text { Atherogenic } \\
\text { coefficient }\end{array}$ & $\begin{array}{l}\text { - atherosclerosis; } \\
\text { - coronary heart disease; } \\
\text { - cardiovascular diseases; }\end{array}$ & $\begin{array}{l}\text { - decreased concentrations of lipoproteins in } \\
\text { blood by right treatment. }\end{array}$ \\
\hline Anti-MCV & $\begin{array}{l}\text { - rheumatoid arthritis; } \\
\text { - other rheumatic diseases. }\end{array}$ & \\
\hline $\begin{array}{l}\text { Total choles- } \\
\text { terol }\end{array}$ & $\begin{array}{l}\text { - coronary heart disease; } \\
\text { - primary dyslipidemia; } \\
\text { - secondary dyslipidemia; } \\
\text { - bile duct obstruction; } \\
\text { - high-fat and -cholesterol diet. }\end{array}$ & $\begin{array}{l}\text { - hypo-, alpha-, beta-lipoproteinemias; } \\
\text { - severe hepatocellular injuries; } \\
\text { - chronic obstructive pulmonary diseases; } \\
\text { - fasting; } \\
\text { - chronic anemias. }\end{array}$ \\
\hline HDL & $\begin{array}{l}\text { - hyper-alpha-lipoproteinemia; } \\
\text { - hyper-beta-lipoproteinemia; } \\
\text { - chronic liver diseases; } \\
\text { - treatment with insulin; } \\
\text { - long-term physical exercises. }\end{array}$ & $\begin{array}{l}\text { - familial hypo-alpha-lipoproteinemia; } \\
\text { - familial hypertriglyceridemia; } \\
\text { - Apo A-I and Apo C-III deficiency; } \\
\text { - chronic kidney disease; } \\
\text { - hepatocellular pathology, cholestasis. }\end{array}$ \\
\hline LDL & $\begin{array}{l}\text { - familial hypercholesterolemia (type IIa) ; } \\
\text { - hyperlipoproteinemias IIb and III; }\end{array}$ & $\begin{array}{l}\text { - hypolipoproteinemias; } \\
\text { - beta-lipoproteinemias; }\end{array}$ \\
\hline
\end{tabular}




\begin{tabular}{|c|c|c|}
\hline & $\begin{array}{l}\text { - high-cholesterol diet; } \\
\text { - chronic renal failure. }\end{array}$ & $\begin{array}{l}\text { - hyperthyreosis; } \\
\text { - chronic anemia; } \\
\text { - acute sepsis; } \\
\text { - inflammatory arthropathies; } \\
\text { - chronic pulmonary diseases. }\end{array}$ \\
\hline AST & $\begin{array}{l}\text { - acute myocardial infarction (in 6-12 h); } \\
\text { - extensive liver necroses; } \\
\text { - viral, toxic hepatitises; } \\
\text { - myositis, myocarditis; }\end{array}$ & $\begin{array}{l}\text { - azotemia; } \\
\text { - chronic dialysis; } \\
\text { - alcoholism. }\end{array}$ \\
\hline ALT & $\begin{array}{l}\text { - myocardial infarction; } \\
\text { - heart failure; } \\
\text { - myositis; } \\
\text { - myocarditis; } \\
\text { - myodystrophy. }\end{array}$ & $\begin{array}{l}\text { - infections of the genitourinary tract; } \\
\text { - alcoholic hepatitis. }\end{array}$ \\
\hline Triglycerides & $\begin{array}{l}\text { - myocardial infarction; } \\
\text { - atherosclerosis; } \\
\text { - renal diseases. }\end{array}$ & $\begin{array}{l}\text { - malabsorption; } \\
\text { - hyperthyroidism; } \\
\text { - chronic obstructive pulmonary diseases. }\end{array}$ \\
\hline Troponin & $\begin{array}{l}\text { - myocardial infarction; } \\
\text { - unstable angina; } \\
\text { - myocardites; } \\
\text { - myocardial injuries after angioplasty, bypass } \\
\text { surgery. }\end{array}$ & - tested in $12 \mathrm{~h}$ after pain syndrome; \\
\hline D-dimer & $\begin{array}{l}\text { - stroke; } \\
\text { - DIC; } \\
\text { - myocardial infarction; } \\
\text { - arterial or venous thromboses; } \\
\text { - renal, hepatic insufficiency; }\end{array}$ & $\begin{array}{l}\text { - lack of tissue plasminogen activator (tPA) } \\
\text { at high plasminogen activator inhibitor (PAI- } \\
\text { I) level; } \\
\text { - small size of a blood clot; } \\
\text { - testing its concentration in } 6 \mathrm{~h} \text { after the } \\
\text { attack.. }\end{array}$ \\
\hline
\end{tabular}

In clinical practice, physicians often meet such concepts as «false-negative» and «false-positive» result, qualitative or semi-quantitative false result. This occurs in the first place because of wrong choice of the method for any marker testing. Therefore, for example, in order to perform monitoring of acute myocardial infarction, it is necessary to test troponin level only quantita- tively, because only in this case it is seen how its numerical indices are changing on the treatment stages. The second and highly meaningful cause of false laboratory index is wrong preparation for collection by the patient. Most patients know that all samples have to be submitted in fasting state, but other conditions influencing their indices additionally are often unknown to them (table 4).

Table 4

Factors that can negatively influence plausibility of laboratory indices submitted by the patient

\begin{tabular}{|c|c|c|}
\hline Indices & Aim of examination & Factors that influence the result \\
\hline LDH & $\begin{array}{l}\text { - prognosis of treatment; } \\
\text { - late diagnostics of myocardial infarction; } \\
\text { - diagnostics of necrosis spread; } \\
\text { - diagnostics of anemias accompanied with hemoly- } \\
\text { sis. }\end{array}$ & $\begin{array}{l}\text { - alcohol, caffeine, aspirin; } \\
\text { - leukemia; } \\
\text { - lymphoma; } \\
\text { - hypothyroidism; } \\
\text { - injuries, fractures; } \\
\text { - megaloblastic anemia; } \\
\text { - hyperthermia; } \\
\text { - infectious mononucleosis }\end{array}$ \\
\hline CPK-МB & $\begin{array}{l}\text { - diagnostics of myocardial infarction; } \\
\text { - cause specification of chest pain syndrome; } \\
\text { - diagnostics of pulmonary embolism; } \\
\text { - diagnostics of dermatomyosites on early stage. }\end{array}$ & $\begin{array}{l}\text { - hemolysis; } \\
\text { - poisoning; } \\
\text { - physical exercise; } \\
\text { - amlodipines; } \\
\text { - bisoprolol; } \\
\text { - lidocaine; } \\
\text { - hypothyroidism; } \\
\text { - Reye syndrome; } \\
\text { - PE, pulmonary edema; } \\
\text { - injuries; } \\
\text { - pregnancy. }\end{array}$ \\
\hline Potassium & $\begin{array}{l}\text { - diagnostics of cardiovascular pathology; } \\
\text { - differential diagnostics of cardiac arrhythmia, arte- }\end{array}$ & $\begin{array}{l}\text { - decomposing of tumors; } \\
\text { - deep burns; }\end{array}$ \\
\hline
\end{tabular}




\begin{tabular}{|c|c|c|}
\hline & $\begin{array}{l}\text { rial hypertension; } \\
\text { - monitoring of potassium levels by intake of diuret- } \\
\text { ics and cardiac glycosides. }\end{array}$ & $\begin{array}{l}\text { - acidosis; } \\
\text { - digoxin; } \\
\text { - NSAIDs; } \\
\text { - adrenal insufficiency. }\end{array}$ \\
\hline Sodium & $\begin{array}{l}\text { - diagnostics of GIT disorders: vomiting, diarrhea; } \\
\text { - diagnostics of adrenal insufficiency. }\end{array}$ & $\begin{array}{l}\text { - ACTH; } \\
\text { - anabolic steroids; } \\
\text { - contraceptives; } \\
\text { - hyperthermia; } \\
\text { - significant dehydration. }\end{array}$ \\
\hline CRP & $\begin{array}{l}\text { - diagnostics of rheumatic diseases; } \\
\text { - diagnostics of myocardial infarction; } \\
\text { - evaluation of inflammation activity grade; } \\
\text { - diagnostics of sepsis, meningitis. }\end{array}$ & $\begin{array}{l}\text { - postsurgical complications; } \\
\text { - secondary amyloidosis; } \\
\text { - tuberculosis. }\end{array}$ \\
\hline $\begin{array}{l}\text { Atherogenic } \\
\text { coefficient }\end{array}$ & - evaluation of treatment of hyperlipoproteinemias. & \\
\hline Anti-MCV & - diagnostics of rheumatic disorders. & \\
\hline $\begin{array}{l}\text { Total choles- } \\
\text { terol }\end{array}$ & $\begin{array}{l}\text { - monitoring of increased risk factors of coronary } \\
\text { heart disease; } \\
\text { - screening of primary and secondary dyslipidemia; } \\
\text { - monitoring of treatment of dyslipidemias. }\end{array}$ & $\begin{array}{l}\text { - hyperthyroidism; } \\
\text { - myeloproliferarative disorders; } \\
\text { - acute inflammatory diseases, infections. }\end{array}$ \\
\hline HDL & $\begin{array}{l}\text { - evaluation of coronary heart disease risk; } \\
\text { - diagnostics of hyperproteinemias; } \\
\text { - diagnostics of atherosclerosis, cardiovascular and } \\
\text { liver diseases. }\end{array}$ & $\begin{array}{l}\text { - contraceptives; } \\
\text { - estrogens; } \\
\text { - phenobarbital; } \\
\text { - captopril; } \\
\text { - furosemide; } \\
\text { - nifedipine; } \\
\text { - diabetes mellitus. }\end{array}$ \\
\hline LDL & $\begin{array}{l}\text { - differentiation of lipoproteinemias; } \\
\text { - diagnostics of atherosclerosis. }\end{array}$ & $\begin{array}{l}\text { - hypothyroidism; } \\
\text { - nephritic syndrome; } \\
\text { - diabetes mellitus; } \\
\text { - anorexia; } \\
\text { - pregnancy. }\end{array}$ \\
\hline AST & $\begin{array}{l}\text { - diagnostics and differential diagnostics of myocar- } \\
\text { dial infarction; } \\
\text { - differentiation of liver pathology. }\end{array}$ & $\begin{array}{l}\text { - opioids; } \\
\text { - pregnancy; } \\
\text { - myodystrophies, } \\
\text { - injuries; } \\
\text { - hypothermia; } \\
\text { - toxic hepatitises. }\end{array}$ \\
\hline ALT & $\begin{array}{l}\text { - monitoring of heart failure; } \\
\text { - provement of MI; } \\
\text { - diagnostics of myocarditis; } \\
\text { - diagnostics of inflectional mononucleosis; }\end{array}$ & $\begin{array}{l}\text { - severe burns; } \\
\text { - shock states; } \\
\text { - hepatitis, cyrhosis; } \\
\text { - liver cancer; } \\
\text { - acute pancreatitis; } \\
\text { - toxicants; }\end{array}$ \\
\hline Triglycerides & $\begin{array}{l}\text { - evaluation of atherosclerosis risk; } \\
\text { - diagnostics of MI; } \\
\text { - diagnostics of familial lipid storage diseases. }\end{array}$ & $\begin{array}{l}\text { - pancreatitis; } \\
\text { - gout; } \\
\text { - overeating; } \\
\text { - obesity; } \\
\text { - sedentary life-style; } \\
\text { - pregnancy. }\end{array}$ \\
\hline Troponin & $\begin{array}{l}\text { - making of MI diagnosis; } \\
\text { - specification of MI dynamics; } \\
\text { - prognosis of MI; } \\
\text { - specification of death risk by MI; }\end{array}$ & $\begin{array}{l}\text { - injuries; } \\
\text { - cardiac or renal decompensation; } \\
\text { - pulmonary embolism; } \\
\text { - acute stroke. }\end{array}$ \\
\hline D-dimer & $\begin{array}{l}\text { - diagnostics of thrombosis; } \\
\text { - monitoring of thrombolytic therapy; } \\
\text { - post-rehabilitation period of patients with stroke }\end{array}$ & $\begin{array}{l}\text { - estrogens; } \\
\text { - severe injuries; } \\
\text { - pregnancy; } \\
\text { - ovarian cancer; } \\
\text { - plasmagene. }\end{array}$ \\
\hline
\end{tabular}


Also, laboratory values may be influenced as by sharp overcooling or overheat (sauna, sun on the beach) the day before, wrong choice of time, day for submitting of hormone panel after 10:00 am for example, as well as intake of some medication groups (table 4).

Neurohormonal markers. HF is accompanied with increasing of various other neurohormonal markers (noradrenalin, renin, aldosterone, endothelin, arginine, and vasopressin). Nevertheless, it has no use in testing, and evaluation of neurohormonal activation for diagnostic or prognostic aim for each patient.

Natriuretic peptides. An important biomarker for diagnostics of $\mathrm{HF}$ and monitoring of patients with established chronic heart failure is testing of natriuretic peptides concentration in plasma.

Normal concentration has a high negative prognostic level in untreated patients and shows that HF is the least probable cause of symptoms in this case. This can play a significant role, especially for primary aid. A high level of natriuretic peptides that is observed regardless of optimal treatment indicates bad prognosis $[3,5]$.
Testing of brain natriuretic peptide (BNP) and its N-terminal fragment (NT-proBNP) was presented as means for diagnostics and management of HF (table 3). They increase in response to higher stretch of myocardial wall. In general, lower levels are seen in patients with preserved left ventricular systolic function. There is no precise limit for both natriuretic peptides used for diagnostics of $\mathrm{HF}$ in the intensive care unit. In relation to a long-term half-life of natriuretic peptides, sudden changes of LV filling pressure may not be presented with fast changes of peptide level. States that may show higher natriuretic peptide levels besides HF are LV hypertrophy, tachycardia, RV overload, myocardial ischemia, hypoxemia, renal dysfunction, old age, cirrhosis of the liver, sepsis, infection. Obesity and treatment may decrease the natriuretic peptide level (table 5).

Leading cardiologists proposed for the practices a scheme of physician's actions, when untreated patients had presented HF symptoms for making of diagnosis (scheme 1).

Interpretation of laboratory tests in patients with $\mathrm{HF}$

Table 5

\begin{tabular}{|l|c|c|}
\hline \multicolumn{1}{|c|}{ Evaluation } & \multicolumn{2}{c|}{ Diagnosis of HF } \\
\cline { 2 - 3 } & Proves in the presence & Denies in norm or in the absence \\
\hline Laboratory examinations & & + \\
\hline High BNP / NT-proBNP level & +++ & +++ \\
\hline Low/normal BNP / NT-proBNP level & + & + \\
\hline Hyponatremia & + & + \\
\hline ST2 & +++ & + \\
\hline CPK & +++ & + \\
\hline Insignificantly high troponin level & + & + \\
\hline
\end{tabular}

Scheme 1

The scheme for making a diagnosis of HF with testing of natriuretic peptides in untreated patients with symptoms indicating $\mathrm{HF}$

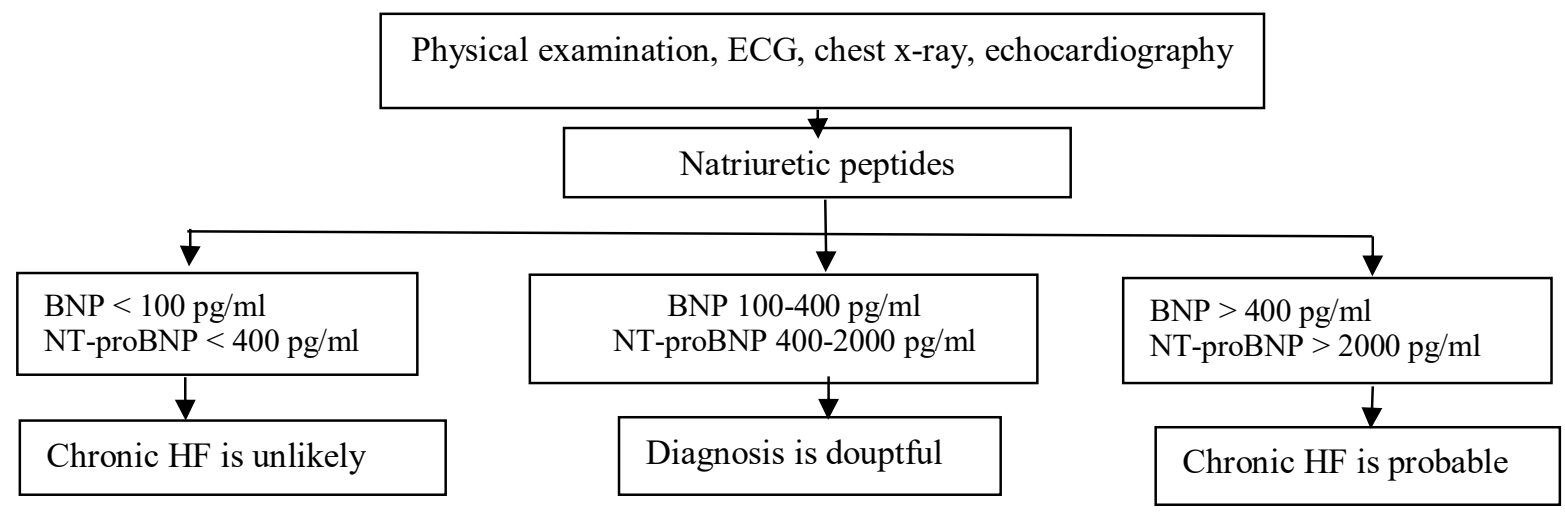

There is evidence-proving effectiveness of natriuretic peptides use for diagnostics, dealing with question considering hospitalization/discharge and specification of individual prognosis, but there is lack of evidence about their use for monitoring and regulation of pharmacological therapy [2].

Heart failure marker - ST2. When natriuretic peptides react to myocyte stretch, then ST2 is a marker of myocardial fibrosis and its remodeling. ST2 is an IL-33 receptor that releases by cardiomyocytes and fibroblasts.

Response of a healthy cardiac tissue to damage or mechanical stress includes production and binding of interleukin-33 (IL-33) with ST2L initializing cardioprotective signaling events for prevention of fibrosis, remodeling of the heart and heart failure (HF). sST2 blocks cardioprotective effect of IL-33 [9]. 
High concentration of circulating in blood ST2 indicates a high risk of adverse effects, hospitalization and even death not only for patients with HF, but also for patients with other CVD forms and in the population at large (table 6). Average normal ST2 concentration is $18 \mathrm{ng} / \mathrm{ml}$; the concentration above $35 \mathrm{ng} / \mathrm{ml}$ indicates a higher risk. Testing of ST2 in patients with HF may lessen risk of their second hospitalization and sudden death. The patients with ST2 $>=35 \mathrm{ng} / \mathrm{ml}$ are administered special treatment that includes monitoring of the patient with administration of additional examinations and diagnostic procedures, medications [9 10].

Table 6

ST2 and NT-proBNP by risk stratification

\begin{tabular}{|l|c|c|c|}
\hline \multicolumn{1}{|c|}{ Category } & Mortality & $\begin{array}{c}\text { Risk level } \\
\text { HR (95\% CI) }\end{array}$ & p \\
\hline ST2 $\leq 35 \mathrm{ng} / \mathrm{ml}$ & $9.0 \%$ & 1 & $<0.001$ \\
NT-proBNP $\leq$ median & $23.3 \%$ & $2.87(1.9-4.32)$ & 0.0115 \\
\hline $\begin{array}{l}\text { ST2 } \leq 35 \mathrm{ng} / \mathrm{ml} \\
\text { NT-proBNP }>\text { median }\end{array}$ & $22.2 \%$ & $2.70(1.25-5.84)$ & $<0.001$ \\
\hline $\begin{array}{l}\text { ST2 }>35 \mathrm{ng} / \mathrm{ml} \\
\text { NT-proBNP } \leq \text { median }\end{array}$ & $38.9 \%$ & $5.59(3.61-8.66)$ & \\
\hline ST2 $>35 \mathrm{ng} / \mathrm{ml}$ & & & \\
NT-proBNP $>$ median & & & \\
\hline
\end{tabular}

Note: Data were taken from an ambulatory cohort of patients with CHF (HF-ACTION trial, USA). Median value for NT-proBNP was $852 \mathrm{pg} / \mathrm{ml}$. Follow-up of the trial was 4 years. (Felker M et al., in Press)

Unique advantages of ST2 marker:

1. It increases several years before cardiac events.

2. It does not depend on renal function.

3. It is a cardiac transplant rejection marker.

Timely test and regular monitoring of ST2 level allows:

1. To prevent heart failure.

2. To decrease second hospitalization risk during 30 days by $17.3 \%$.

3. To decrease sudden death risk during 30 days by $17.6 \%$.

ST2 testing by discharge allows decreasing significantly rehospitalization cases during 30 days. BNP level by ST2 $>35 \mathrm{ng} / \mathrm{ml}$ may be as higher, as well as normal that can disorient physicians (scheme 2.).

\section{Management of patients with heart failure.}

Scheme 2

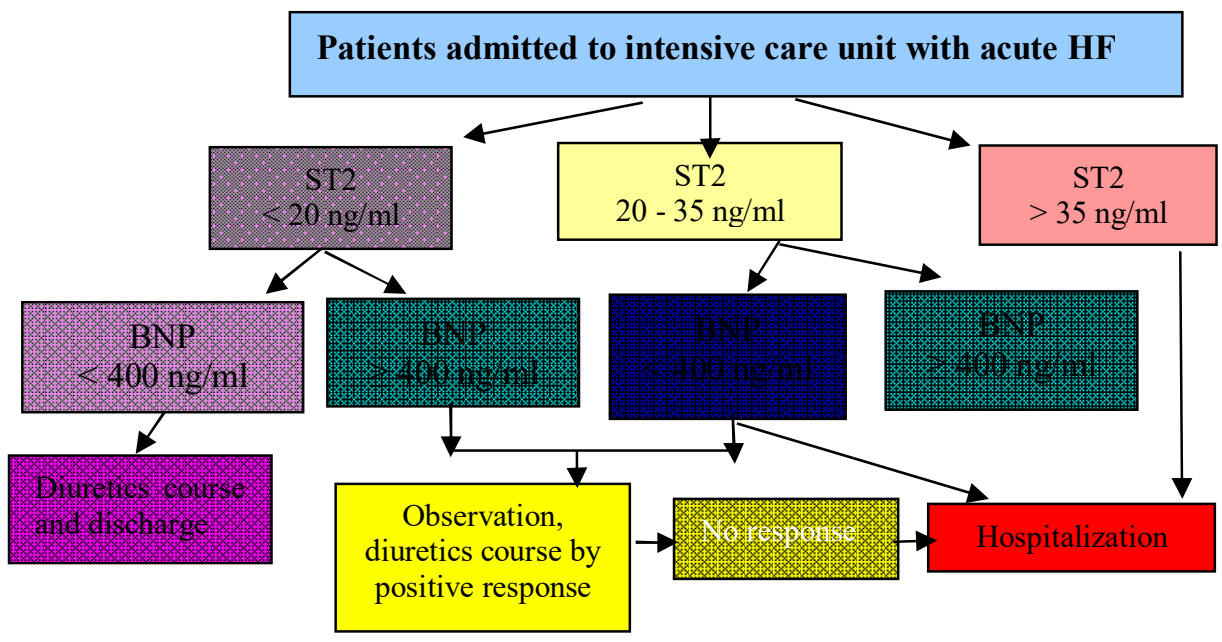

According to statistics:

- patients with HF have significantly increased morbidity and mortality rate after the discharge from the hospital $\left(40 \%\right.$ of patients die during the $1^{\text {st }}$ year after the discharge from the hospital);

- second hospitalization of patients with acute decompensate heart failure (ACHF) is $25 \%$ during 30 days and $70 \%$ during 1 year and significantly correlates with increased mortality rate during the $1^{\text {st }}$ year.

Back in 1933, Sir Thomas Lewis wrote in his book about cardiac diseases : «The very essence of cardiovascular practice is the early detection of heart failure» [15]. After all, the second hospitalization and early complications may be avoided providing development of evaluation methods and choice of therapy for patients 
with HF. One of such methods is an early heart failure marker ST2.

\section{Conclusions:}

1. As ST2 does not depend on such factors like BMI, gender, age, smoking status, comorbid pathologies (mainly liver dysfunction), it may be one of the «strongest» prognostic HF markers.

2. Introduction of ST2 into routine practice will allow decreasing significantly expenses of the hospitals (decreasing amount of second hospitalizations, unnecessary drug withdrawal etc.) and improving life quality of the patients with $\mathrm{HF}$ (decreasing progress speed of HF).

3 . Based on stated above we can precisely tell that the earliest diagnostic marker of heart failure is ST2 marker.

4. Only complex examination can show a complete characteristics of myocardium functional state, so combined use of several HF markers is most acknowledged in the whole world, precisely the multimarker model - NP + ST2 (TnI).

\section{References:}

1. Voronkov LH, Parascheniuk LP. Peryferychna miopatiya yak terapevtychna «mishen» pry khronichniy sertseviy nedostatnosti. Sertseva nedostatnist ta komorbidni stany. 2017; 2:47-53.

2. Rekomendatsiyi $\mathrm{z}$ diahnostyky ta likuvannia hostroyi ta khronichnoyi sertsevoyi nedostatnosti Yevropeyskoho Tovarystva Kardiolohiv. Dosvid ta dokazy. 2008; 8(54):19-28.

3. Kishkun AA. Rukovodstvo po laboratornym metodam diagnostiki. Moscow: GEOTAR-Media; 2014. P.760.

4. Seredyuk NM, Kuryliv HM. Korektsiya klinichnykh proyaviv khronichnoyi sertsevoyi nedostatnosti u khvorykh iz perenesenym infarktom miokarda. Galician Medical Journal. 2013, May; 2(20):105-8.

5. Titov VN. Laboratornye i instrumentalnye issledovaniya v diagnostike: Spravochnik. Moscow; GEOTARMED; 2004. P.960.

6. WHO [Internet]. Geneva: WHO; c2005-2019. WHO Mortality Database [cited 2019 Feb 6]; [about 1 screen]. Available

from:

http://www.who.int/healthinfo/mortality_data/en

7. World Heart Federation [Internet]. Geneva: WHF; 2017. World Heart Day [cited 2019 Feb 6]; [about 6 screens]. Available from: https://www.world-heartfederation.org/world-heart-day/resources.

8. State Statistics Service of Ukraine. Healthcare. [Internet]. Kyiv: State Statistics Service of Ukraine; 2016 [updated 2017 Jul 12; cited 2019 Feb 6]. Available from: http://www.ukrstat.gov.ua.

9. Zhyvy sertsem [Internet]. Kyiv: Center for Pediatric Cardiology and Cardiac Surgery; c2005-2018. Zhyvy sertsem [cited 2019 Jan 14]; [about 2 screens]. Available from: http://zhyvy-sercem.com.ua.

10. Huang J, Yang J, Zhang J. Chinese guidelines for the diagnosis and treatment of heart failure. Chin J Cardiol. 2014, feb; 42(2):98-122.

11. Mueller-Hennessen M, Sigl J, Fuhrmann Jc, Witt H, Reszka R, Schmitz O, et al. Metabolic profiles in heart failure due to non-ischemic cardiomyopathy at rest and under exercise. Esc heart fail. 2017 may; 4(2):178-189.
12. Maeyer C, Beckers P, Vrints CJ, Conraads VJ. Exercise training in chronic heart failure. Ther Adv Chronic Dis. 2013, May; 4(3):105-117.

13. Lavie CJ, Milani RV, Ventura HO. Adipose composition and heart failure prognosis. J Am Coll Cardiol 2017; 70:2750-1.

14. Haas M, Zugck C, Kubler W. Der 6-minuten-gehtest: eine kostengünstige Alternative zur Spiroergometrie bei Patienten mit chronischer Herzinsuffizienz? Zeitschrift für Kardiologie. 2000, Feb; 2(89):72-80.

15. Ponikowski P, Voors AA, Anker SD, Bueno H, Cleland JGF, Coats AJS et al. 2016 ESC Guidelines for the diagnosis and treatment of acute and chronic heart failure: The Task Force for the diagnosis and treatment of acute and chronic heart failure of the European Society of Cardiology (ESC)Developed with the special contribution of the Heart Failure Association (HFA) of the ESC. Eur Heart J. 2016, Jul 14; 37(27):2129-2200.

\section{УДК 616-072+616.12-008.46 \\ ЛАБОРАТОРНА ДІАГНОСТИКА РАННЬОЇ СЕРЦЕВОЇ НЕДОСТАТНОСТІ}

\author{
Н.Б. Тимочко
}

\author{
Івано-Франківський начіональний медичний \\ університет, кафедра внутрішньої медицини №2 \\ та медсестринства, \\ м. Івано-Франківськ, Украӥна, \\ ORCID ID: 0000-0002-5319-5468, \\ e-mail:dr.ntymochko@gmail.com
}

Резюме. Багато захворювань серцевосудинної системи потребують саме своєчасної діагностики, щоб запобігти гострих та хронічних патологічних процесів і ускладнень. У статті представлені лабораторні показники та маркери, використання яких допомагає сучасному лікарю правильно та своєчасно поставити діагноз, спрогнозувати виникнення у пацієнтів хворіб та моніторингу лікування такого діагнозу як серцева недостатність. Існують докази, що підтверджують ефективність використання натрійуретичних пептидів для діагностики, вирішення питання щодо госпіталізації / виписки і визначення індивідуального прогнозу, а от доказів щодо їх застосування для спостереження та регулювання медикаментозної терапії бракує. Відповідь здорової серцевої тканини на пошкодження або механічний стрес включає продукцію та зв'язування інтерлейкіну-33 (IL-33) з ST2L, запускаючи кардіозахисний сигнальний каскад запобігання фіброзу, ремоделювання серця та серцевої недостатності $(\mathrm{CH})$. sST2 блокує кардіопротектівним ефект IL-33. Визначення ST2 при виписці дозволяє суттєво скоротити випадки регоспіталізації протягом 30 днів. Рівень BNP при ST2 >35 нг/мл як підвищеним, так і нормальним, що може ввести в оману лікарів.

Оскільки ST2 не залежить від таких факторів як IMT, стать, вік, статус паління, наявність супутніх патологій, він може бути одним із найбільш «сильних» прогностичних маркерів СН. Введення 
ST2 в рутинну практику дозволить суттєво скоротити видатки лікарень (зниження кількості повторних госпіталізацій, відміна непотрібних препаратів тощо) і підвищити якість життя пацієнтів з СН (знизити швидкість прогресування СН). На основі вище зазначеного, можна точно сказати, що найбільш раннім діагностичним маркером серцевої недостатності $\epsilon$ маркер - ST2.

Ключові слова: лабораторні методи діагностики, серцева недостатність, маркер серцевої недостатності, ST-2.

\section{УДК 616-072+616.12-008.46 \\ ЛАБОРАТОРНАЯ ДИАГНОСТИКА РАННЕЙ СЕРДЕЧНОЙ НЕДОСТАТОЧНОСТИ}

\section{Н.Б. Тимочко}

Ивано-Франковский национальный медицинский университет, кафедра внутренней медицины №2

и медсестринства,

2. Ивано-Франковск, Украина,

ORCID ID: 0000-0002-5319-5468,

e-mail:dr.ntymochko@gmail.com

Резюме. Многие заболевания сердечнососудистой системы нуждаются именно в своевременной диагностике, чтобы предотвратить возникновение острых и хронических патологических процессов и осложнений. В статье представлены лабораторные показатели и маркеры, использование которых помогает современному врачу правильно и своевременно поставить диагноз, спрогнозировать возникновения у пациентов болезней и мониторинга лечения такого диагноза, как сердечная недостаточность. Существуют доказательства, подтверждающие эффективность использования натрийуретических пептидов для диагностики, решения вопроса о госпитализации / выписки и определения индивидуального прогноза, а вот доказательств их применения для наблюдения и регулирования медикаментозной терапии не хватает. Ответ здоровой сердечной ткани на повреждение или механический стресс включает продукцию и связывания интерлейкина-33 (IL-33) с ST2L, запуская кардиозащитный сигнальный каскад предотвращения фиброза, ремоделирования сердца и сердечной недостаточности (CH). sST2 блокирует кардиопротективным эффект IL-33. Определение ST2 при выписке позволяет существенно сократить случаи регоспитализации в течение 30 дней. Уровень BNP при ST2 >35 нг / мл как повышенным, так и нормальным, что может ввести в заблуждение врачей.

Поскольку ST2 не зависит от таких факторов как ИМТ, пол, возраст, статус курения, наличие сопутствующих патологий, он может быть одним из самых «сильных» прогностических маркеров $\mathrm{CH}$. Введение ST2 в рутинную практику позволит существенно сократить расходы больниц (снижение количества повторных госпитализаций, отмена ненужных препаратов и т.д.) и повысить качество жизни пациентов с СН (снизить скорость прогрессирования $\mathrm{CH}$ ). На основе выше сказанного, можно точно сказать, что самым ранним диагностическим маркером сердечной недостаточности является маркер - ST2.

Ключевые слова: лабораторные методы диагностики, сердечная недостаточность, маркер сердечной недостаточности, ST-2.

Стаття надійшла в редакцію 09.03.2019 р. 